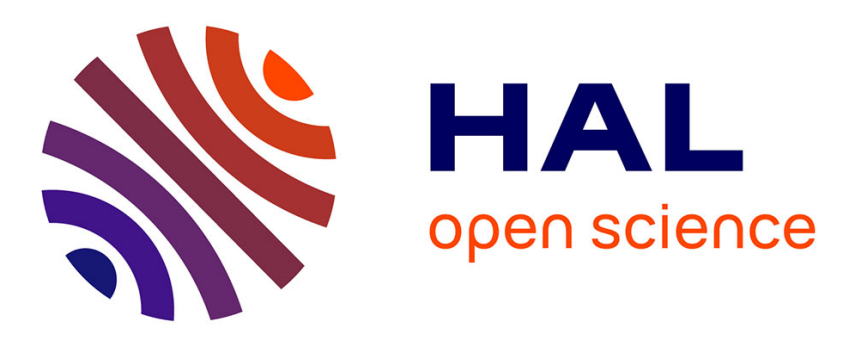

\title{
Spin orbit torque non-volatile flip-flop for high speed and low energy applications
}

Kotb Jabeur, Gregory Di Pendina, Fabrice Bernard-Granger, Guillaume Prenat

\section{- To cite this version:}

Kotb Jabeur, Gregory Di Pendina, Fabrice Bernard-Granger, Guillaume Prenat. Spin orbit torque non-volatile flip-flop for high speed and low energy applications. IEEE Electron Device Letters, 2014, 35 (3), pp.408 - 410. 10.1109/led.2013.2297397 . hal-03506992

\section{HAL Id: hal-03506992 https://hal.science/hal-03506992}

Submitted on 12 Jan 2022

HAL is a multi-disciplinary open access archive for the deposit and dissemination of scientific research documents, whether they are published or not. The documents may come from teaching and research institutions in France or abroad, or from public or private research centers.
L'archive ouverte pluridisciplinaire HAL, est destinée au dépôt et à la diffusion de documents scientifiques de niveau recherche, publiés ou non, émanant des établissements d'enseignement et de recherche français ou étrangers, des laboratoires publics ou privés. 


\title{
Spin orbit torque non-volatile flip-flop for high speed and low energy applications
}

\author{
K. Jabeur, G. Di Pendina, F. Bernard-Granger, G. Prenat
}

\begin{abstract}
A novel non-volatile flip-flop based on spin-orbit torque magnetic tunnel junctions (SOT-MTJ) is proposed for fast and ultra-low energy applications. A case study of this nonvolatile flip-flop is considered. In addition to the independence between writing and reading paths which offers a high reliability, the low resistive writing path performs high speed and energyefficient write operation. We compare the SOT-MTJ performances metrics with the spin transfer torque (STT-MTJ). Based on accurate compact models, simulation results show an improvement which attains $20 \times$ in terms of write energy per bit cell. At the same writing current and supply voltage, the SOTMTJ achieves a writing frequency $4 \times$ higher than the STT-MTJ.
\end{abstract}

Index Terms - Spin orbit torque, Spin transfer torque, MRAM, Spintronic, spin hall effect, three-terminal

\section{INTRODUCTION}

Spin transfer torque magnetic random access memory (STT-MRAM) [1] is a giant step toward a "universal memory" which could replace all conventional memory types while gathering their most merits; speed, high density, cost benefits, endurance and Non-Volatility. However, two main shortcomings are still limiting the reliability and endurance of STT-MRAMs; i) The high current density required for writing can occasionally damage the MTJ barrier, ii) It remains a challenge to fulfil a reliable reading without ever causing switching. Because writing and reading operations share the same path (through the junction), more constraints limit the designer to achieve high speed and reliable MRAM architectures. The SOT-MRAM is a promising alternative for the STT-MRAM. Thanks to its three-terminal architecture, authors in [2-5] claim that SOT-based devices can be built with independent reading and writing paths and so avoid challenges encountered with the state of the art of conventional STTMRAMs, while keeping competitive switching currents. A thrilling debate is in progress between research groups inquiring about the exact origins of the SOT switching mechanism. It is still unobvious whether the Rashba effect has a significant role associated to the Spin Hall Effect SHE to achieve the switching [2-3] or it is only due to the SHE [4-5]. That's why we use the abbreviation "SOT" to design this innovative device, since this term reveals the spin-orbit-torque responsible for switching with taking into account both phenomena, Rashba and SHE.

In this letter, we highlight the capability of the SOT device to design low energy and high speed MRAM architectures.

The work and results reported were obtained on the framework of the spOt project (grant agreement $n^{\circ} 318144$ ) funded by the European Commission under the Seventh Framework Programme. All authors are with SPINTEC Lab, CEA Grenoble, France (17 rue des Martyrs 38054 Grenoble Cedex 9). K. Jabeur (kotb.jabeur@cea.fr), G.Di Pendina (gregory.dipendina@cea.fr), F. Bernard-Granger (fabrice.bernardgranger@cea.fr) and G. Prenat (guillaume.prenat@cea.fr).
An example of a non volatile flip flop (NVFF) is studied. NVFF can provide a more efficient use of energy in systems on chips for standby-power-critical and quick-startup applications such as battery operated appliances and also improve performance of VLSI circuits [6]. Simulation results emphasize the improvement achieved with the SOT-MTJ when compared to the STT-MTJ. Both technologies considered in this letter are perpendicular magnetic anisotropy (PMA) materials-based. For simulation results, spice-like compact models -developed in Verilog-A language- have been used for each technology. Both STT and SOT compact models are developed in SPINTEC Lab according to the same magnetic assumptions.

\section{SOT-MTJ STRUCTURE}

A representative geometry of a 3-terminal memory cell with the perpendicular switching of the magnetized ferromagnetic layer using the SOT writing mechanism is shown in figure 1. Figures 1(b) and 1(c) represent the independent writing and reading paths, respectively. The structure consists of a free layer nanomagnet on the top of a writing stripe (metal electrode). For clarity reasons, we present only the main layers of the MTJ stack. Additional layers and materials combination could be considered to increase the robustness of the whole structure and mitigate undesirable effects (e.g. dipole fields around the free layers, etc). For instance, a more complex and complete structure has been proposed in [5]. The writing electrode materials existing in literature to fabricate the sample device are: $\beta$-Tantalum $(\beta$ Ta), $\beta$-Tungsten $(\beta-\mathrm{W})$ or Pt [2-5]. The magnetic cell is written by applying a charge current via the write line. The orientation of the storage layer magnetization is controlled by the direction of the applied charge current. Positive currents (along $+x$ ) produce a spin injection current with transport direction (along $+\mathrm{z}$ ) and spins pointing to $+\mathrm{y}$ direction. The injected spin current in-turn produces spin torque to align the magnet in the $+y$ or $-y$ direction. An external magnetic field is permanently applied to complete the switching operation. More details about the SOT-MTJ behavior are available in [2-5] [7-8].
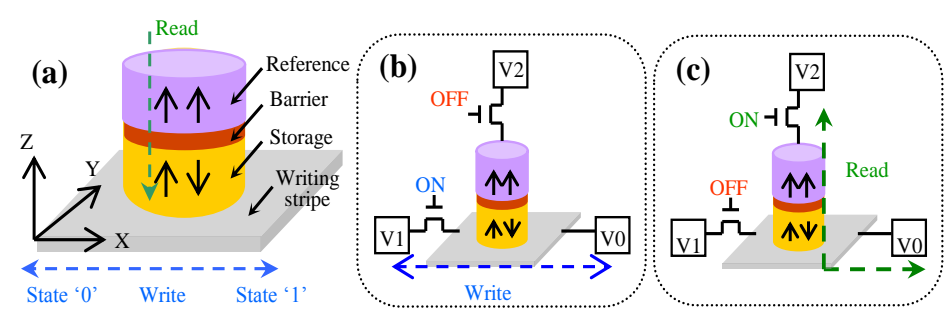

Fig. 1. (a) Schematic of the three-terminal SOT device and the two independent paths for (b) write and (c) read operations. In-plane current injection through the write line (writing stripe) induces the perpendicular switching of the storage layer. 


\section{SOT-MTJ MODELING AND SIMULATION}

For the design of "hybrid CMOS" integrated circuits (ICs) including magnetic devices, it is important to use an accurate compact model of the SOT-MTJ. In [7], a compact model written in Verilog-A of a SOT device has been described and two writing schemes have been discussed in [8]. The model is developed under macrospin approximation and describes straightforward, high-speed and precise electrical representation of the physical behavior of the SOT-MTJ. Based on experiments and characterization of the device samples [23], a number of associated parameters were fed into these equations. The simulation parameters are listed in table I.

The model describes the memory cell as a three-terminal logic device and includes the dynamic behavior described by the Landau-Lifshitz-Gilbert model (LLG) given in equation (1) [9]. A special interest has been given to Rashba effect and SHE torques integrated in the effective magnetic field in equation (2) inside the LLG model to highlight the impact of these two factors on the dynamic of magnetization switching intensively argued in [2-5].

$\frac{\partial \vec{m}}{\partial t}=-\frac{\gamma_{0}}{1+\alpha^{2}}\left(\vec{m} \times \vec{H}_{e f f}\right)-\alpha \frac{\gamma_{0}}{1+\alpha^{2}} \vec{m} \times\left(\vec{m} \times \vec{H}_{e f f}\right)(1)$

$\vec{H}_{e f f}=\vec{H}_{R}+\vec{H}_{S H E}+\vec{H}_{k}+\vec{H}_{a}+\vec{H}_{d}(2), \mathrm{H}_{\mathrm{R}}$ is the Rashba field, $\mathrm{H}_{\text {SHE }}$ is the SHE field, $\mathrm{H}_{\mathrm{k}}$ is the magnetocrystalline anisotropy field, $\mathrm{H}_{\mathrm{a}}$ is the external bias magnetic field and $\mathrm{H}_{\mathrm{d}}$ is the demagnetizing field. Expressions of each magnetic field are:

$\vec{H}_{R}=C_{R} J_{a p p} \vec{y}, H_{S H E}=-C_{S H E} J_{a p p} \vec{m} \times \vec{y}, \vec{H}_{k}=\frac{2 K_{u}}{\mu_{0} M_{s}} m_{z} \vec{z}, \vec{H}_{a}=H_{a} \vec{x}$, $\vec{H}_{d}=-M_{s}\left(n_{x x} \vec{m}_{x}+n_{y y} \vec{m}_{y}+n_{z z} \vec{m} z\right)$

To follow the variation of the SOT-MTJ resistance, Julière's model [10] as well as Simmons's model [11] were used in the expression describing the conductance through the junction. Moreover, for an improved accuracy, we integrated the dynamic conductance given by Brinkman model [12] and we took in consideration the dependence of magneto-resistance on bias voltage. By combining formulas given by these complementary models, we can assume that the total tunnelling conductance can be expressed according to the
TABLE I

DEVICE MODEL PARAMETERS

\begin{tabular}{lll}
\hline \hline Parameter & SOT device & STT device \\
\hline Permeability $\mu_{0}$ & $4 \pi \times 10^{-7}{\mathrm{H} . \mathrm{m}^{-1}}^{3}$ & $4 \pi \times 10^{-7} \mathrm{H} / \mathrm{m}$ \\
Storage layer volume & $40 \times 40 \times 2\left(\mathrm{~nm}^{3}\right)$ & $40 \times 40 \times 2\left(\mathrm{~nm}^{3}\right)$ \\
Writing stripe :dimensions[5] & $(100 \mathrm{~nm}, 50 \mathrm{~nm}, 2.5 \mathrm{~nm})$ & - \\
Resistivity(W, Ta, Pt) [5][13] & $(200,190,20) \mu \Omega . \mathrm{cm}$ & \\
SH angle $(\mathrm{W}, \mathrm{Ta}, \mathrm{Pt})[5][13]$ & $(0.3,0.12,0.08)$ & - \\
Sat. magnetization ,Ms & $1.1 \times 10^{6} \mathrm{~A} / \mathrm{m}$ & $1.1 \times 10^{6} \mathrm{~A} / \mathrm{m}$ \\
Bias magnetic field [3] & $\mathrm{Ha}=5 \times 10^{-3} / \mu_{0}$ & - \\
Thermal stability factor & 104 & 104 \\
Parallel resistance $\mathrm{R}_{\mathrm{P}}$ & $\sim 5 \mathrm{~K} \Omega$ & $\sim 5 \mathrm{~K} \Omega$ \\
Tmr0 & $100 \%$ & $100 \%$ \\
CMOS technology/Vdd & $40 \mathrm{~nm} / 1 \mathrm{~V}$ & $40 \mathrm{~nm} / 1 \mathrm{~V}$ \\
Ic & $60 \mu \mathrm{A}(250 \mathrm{ps})$ & $60 \mu \mathrm{A}(1 \mathrm{~ns})$ \\
$\mathrm{V}_{\text {write }}$ & $1 \mathrm{~V}$ & $1 \mathrm{~V}$ \\
Writing transistors width & $220 \mathrm{~nm}$ & $480 \mathrm{~nm}$
\end{tabular}

equation(3):

$$
g\left(V, m_{x}, m_{z}\right)=\frac{G_{P 0}\left(1-2 \beta V+3 \delta V^{2}\right)}{1+\left(\frac{1-\left(m_{x} \cos \theta_{m h l}+m_{z} \sin \theta_{m h l}\right)}{2}\right)\left(\frac{t m r_{0}}{1+\frac{V^{2}}{V_{h}^{2}}}\right)}
$$

Figure 2(a) describes the theoretical switching of the magnetization $\mathrm{m}_{\mathrm{z}}$ from parallel ' $\mathrm{P}$ ' to 'AP' and vice versa depending on the current direction applied during the time. Figure 2(b) shows the simulation results of the SOT-MTJ model which obviously corresponds to the theoretical behavior of the device and where we clearly observe the oscillations (from the LLG equations) during the switching (write phase).

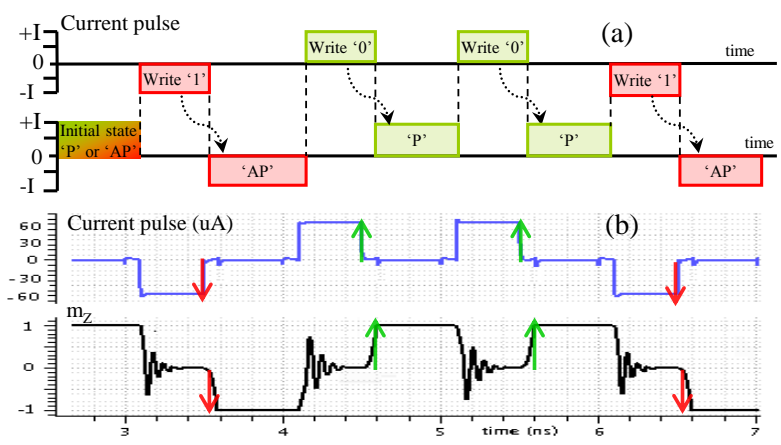

Fig. 2. SOT-MTJ model validation (a) Theoretical behavior (b) model behavior

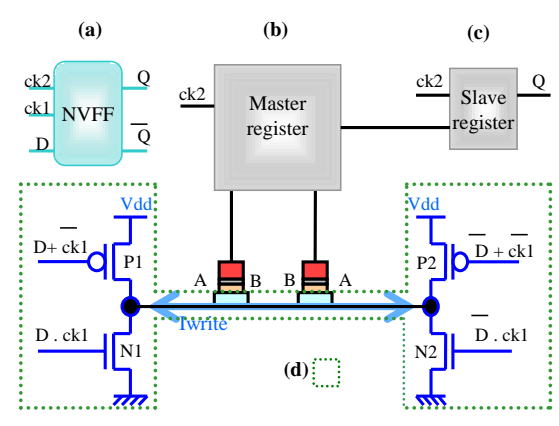

Fig. 3. NVFF structure (a) symbol, (b) master register architecture (c) slave register, (d) writing circuit

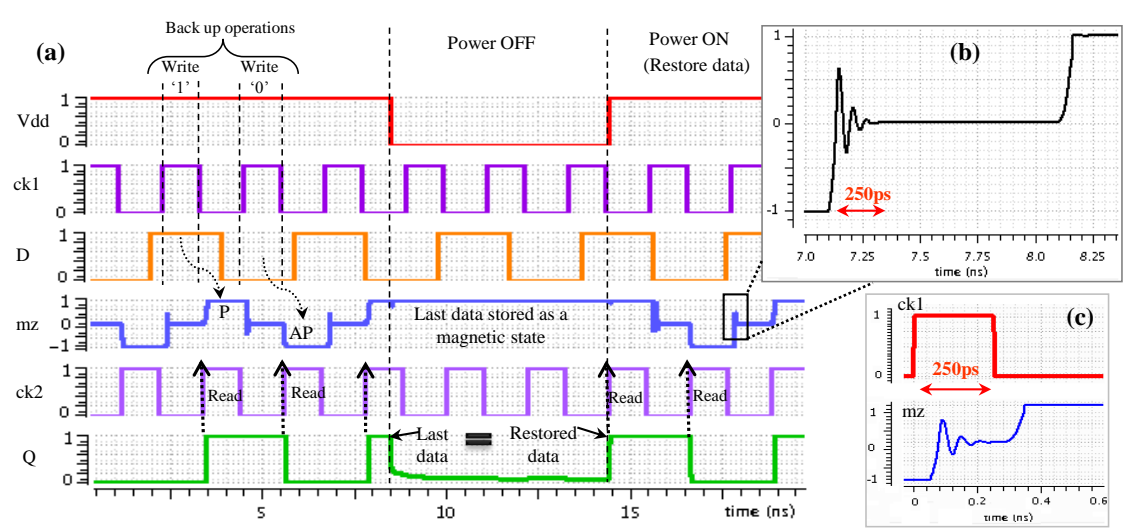

Fig. 4. NVMFF simulation results a) store and restotr operations (b) perpendicular magnetization ' $m z$ ' behavior of the SOT-MRAM during a current pulse of $1 \mathrm{~ns}$ to switch from 'AP' (mz=-1) to ' $\mathrm{P}$ ' $(\mathrm{mz}=1)(\mathrm{c})$ Switching with a $250 \mathrm{ps}$ pulse (exactly after oscillations stabilization) 


\section{RESULTS AND DISCUCCION}

In order to highlight the wide interest of using SOTMRAM, we describe the integration of such device in the architecture of a non volatile magnetic flip flop (NVMFF). This flip flop can be used as a primitive cell into the ASIC design library allowing non volatility, high speed and low power. Figure 3 (a) shows the symbol of the NVMFF. The master register, shown in figure 3(b), is a conventional twoinverters-based non volatile latch based on the same structure in [14]. Its output is connected to the slave latch in figure 3(c). A non-overlapping two-phase clock [15] is used for the design of the NVMFF. The first phase 'ck1' is the writing phase which writes the input data ' $D$ '. It is correlated with the signal 'D' to write its value in the two SOT-MRAMs in a differential way; For instance, when $\mathrm{P} 1$ an $\mathrm{N} 2$ are $\mathrm{ON}$, a path from $\mathrm{V}_{\mathrm{dd}}$ to $\mathrm{g}_{\text {nd }}$ is created and the current flows from A to B for the $1^{\text {st }}$ device and from $\mathrm{B}$ to $\mathrm{A}$ for the $2^{\text {nd }}$ device. While the differential structure is achieved by placing two SOT devices side by side, it is possible to realize a differential structure by stacking them in the same manner in [13]. The second phase clock 'ck2' is the reading phase which reads the magnetic data stored during the writing phase. As shown in figure 4, the output value ' $Q$ ' of the slave register load the magnetic data at each rising edge of the phase clock 'ck2'.

Using the parameters in table I, the critical current required to write an STT-MRAM during a current pulse of $1 \mathrm{~ns}$ is $\mathrm{I}_{C}$ $=60 \mu \mathrm{A}$. Simulation results show that for the same $\mathrm{I}_{\mathrm{C}}$ it is possible to write the SOT-MRAM with only a 250 ps current pulse. Figure 4(b) shows that after 250ps the magnetization $\mathrm{m}_{\mathrm{z}}$ of the free layer gives up the oscillation phase and switching becomes possible. This is validated through simulations, as shown in figure $4(\mathrm{c})$. Thus, the SOT-MRAM is $4 \mathrm{X}$ faster than the STT-MRAM in term of possible writing speed.

In addition, the writing path of the SOT-MRAM is greatly less resistive than the STT-MRAM writing path (through the junction). For example, in our case the equivalent writing path resistance of the two differentials SOT-MRAM is $\mathrm{R}_{\text {eq }}(\mathrm{SOT})=2 \times 1.5 \mathrm{~K} \Omega=3 \mathrm{~K} \Omega$, while for the STT-RAM it is $\mathrm{R}_{\mathrm{eq}}(\mathrm{STT})=\mathrm{R}_{\mathrm{P}}+\mathrm{R}_{\mathrm{AP}}=5 \mathrm{k} \Omega+10 \mathrm{k} \Omega=15 \mathrm{~K} \Omega$. That's why, to drive a writing current of $60 \mu \mathrm{A}$ through the STT-MRAM pair, we needed a $480 \mathrm{~nm}$ width for writing transistors compared to only $220 \mathrm{~nm}$ width in the case of the SOT-MRAM pair. Here, we suppose that we used the same transistor width for $n$ and $p$ type transistors of the writing circuits (figure 3(d)). Moreover, for a 1 ns write time, the SOT-MRAM achieves write energy of 10.8 $\mathrm{fJ}$, which is $5 \times$ smaller than that of the STT-MRAM (54 fJ). Since it is possible to write during only $250 \mathrm{ps}$, the write energy can attain a further improvement of $20 \times$. These orders of magnitude are consistent with the predictive study of SOTMRAM performance in [5]. More performance improvement can be attained with dimensional scaling thanks to a higher spin injection and a lower resistive write path. While, we run our simulation with a supply voltage of $1 \mathrm{~V}$, authors in [5] claim that at low voltage an improvement of more than $100 \times$ can be achieved in term of energy-delay. In the case of the NVFF, there is not any penalty in term of area when we use the SOT device instead of the STT. The writing operation is differential, so the two SOT-MTJs share the same write line and the ' $\mathrm{B}$ ' terminal (figure 3.d) is common. Thus, the two SOT devices could be considered as two-terminal devices during the writing operation. However, in applications such as
TABLE II

TECHNOLOGIES COMPARISON

\begin{tabular}{|c|c|c|c|c|}
\hline & SRAM & FeRAM & $\begin{array}{l}\text { STT- } \\
\text { MRAM }\end{array}$ & $\begin{array}{l}\text { SOT- } \\
\text { MRAM }\end{array}$ \\
\hline Non volatility & No & Yes & Yes & Yes \\
\hline Speed & Fastest & Medium & Fast & Very fast \\
\hline Endurance & $>10^{15}$ & $<10^{12}$ & $>10^{15}$ & $>10^{15}$ \\
\hline Density & Medium & High & High & High \\
\hline Scalability & Good & Limited & Good & Good \\
\hline Standby current & Low/high & Zero & Zero & Zero \\
\hline Process compatiblity & High & Low & High & High \\
\hline Maturity & Product & Product & $\begin{array}{l}\text { Advanced } \\
\text { development }\end{array}$ & $\begin{array}{l}\text { Early } \\
\text { development }\end{array}$ \\
\hline
\end{tabular}

memory arrays, SOT based architecture could be less efficient in term of density because it requires more transistors to separate the reading and the writing operations. In table II, we give an overview about the SOT technology features compared to current technologies and the STT-MRAM.

\section{CONCLUSION}

We propose a NVMFF as a memory application of a three terminal SOT-MRAM where non volatility is achieved with an increased reliability thanks to a separated write/read paths. In addition, the write operation performs $4 \times$ faster than the STTMRAM with an improvement of $20 \times$ in term of writing energy. Therefore, 3-terminal SOT-MRAM is suitable for high speed and low energy applications in addition to its intrinsic reliability and robustness.

\section{REFERENCES}

[1] H. Zhao et al, "A Scaling Roadmap and Performance Evaluation of InPlane and Perpendicular MTJ Based STT-MRAMs for High-Density Cache Memory", IEEE journal of Solid-State Circuits, Vol.48, No. 2, February 2013

[2] P. Gambardella and I. M. Miron, "Current-induced spin-orbit torques", Phil. Trans. R. Soc. A 2011 369, 3175-3197

[3] I.M. Miron et al, "Perpendicular switching of a single ferromagnetic layer induced by in-plane current injection", Nature 476, 189-193, August 2011

[4] Luqiao Liu et al. "Spin-Torque Switching with the Giant Spin Hall Effect of Tantalum", Science 336, 555 (2012)

[5] S. Manipatruni, D.E. Nikonov, I.A. Young, "Voltage and Energy-Delay Performance of Giant Spin Hall Effect Switching for Magnetic Memory and Logic", Ithaca, NY, USA: Cornell Univ. Press, Jan 2013, pp1-16

[6] N. Sakimura et al, "Nonvolatile Magnetic Flip-Flop for Standby-PowerFree SoCs", IEEE journal of solid-state circuits, vol. 44, no. 8, 2009

[7] K. Jabeur et al, "Compact modeling of a Magnetic Tunnel Junction based on Spin Orbit Torque", ISCDG, Dresde, Germany, Sept 2013

[8] K. Jabeur et al, "Study of Two Writing Schemes for A Magnetic Tunnel Junction Based On Spin Orbit Torque", ICCDS, Malaysia, August 2013

[9] L. Landau and E. Lifshitz,'Theory of the dispertion of magnetic permeability in ferromagnetic bodies". Phys. Z. Sowjetunion, 8:153169,1935

[10] M. Julliere, "Tunneling between ferromagnetic films". Phys. Lett. A, 54:225-226, 1975.

[11] J.G Simmons. Generalized formula for the electric tunnel effect between similar electrodes separated by a thin insulating film. J. Appl. Phys., 34:1793-1803, June 1963.

[12] W.F. Brinkman, R.C. Dynes, and J.M. Rowell, "Tunneling conductance of asymmetrical barriers". J. Appl. Phys., 41:1915-1921, April 1970.

[13] Y. Kim et al, "DSH-MRAM: Differential Spin Hall MRAM for On-Chip Memories", IEEE electron device letters, vol. 34, no. 10, 2013

[14] Y. Zhang et al, "Compact Modeling of Perpendicular-Anisotropy $\mathrm{CoFeB} / \mathrm{MgO}$ Magnetic Tunnel Junctions", IEEE transactions on electron devices, vol. 59, no. 3, march 2012

[15] M. Karlsson et al, "A non-overlapping two-phase clock generator with adjustable duty cycle”, Linköping Conference Proceedings, 2003 